\title{
Erratum to: Treatment of the infrared contribution: NLO QED evolution as a pedagogic example
}

\author{
E.G. de Oliveira ${ }^{1,2, a}$, A.D. Martin ${ }^{1}$, M.G. Ryskin ${ }^{1,3}$ \\ ${ }^{1}$ Institute for Particle Physics Phenomenology, University of Durham, DH1 3LE Durham, UK \\ ${ }^{2}$ Instituto de Física, Universidade de São Paulo, C.P. 66318, 05315-970 São Paulo, Brazil \\ ${ }^{3}$ Petersburg Nuclear Physics Institute, NRC Kurchatov Institute, Gatchina, 188300 St. Petersburg, Russia
}

Published online: 16 November 2013

(C) Springer-Verlag Berlin Heidelberg and Società Italiana di Fisica 2013

Erratum to: Eur. Phys. J. C (2013) 73:2534

DOI 10.1140/epjc/s10052-013-2534-3

The final conclusion of this paper is not correct. It was based on our misunderstanding of the treatment of the subtraction term to the NLO ladder diagram given in column 4 of Table 1 of the original calculation of Curci, Furmanski and Petronzio [11]. Contrary to our treatment, Curci et al. do not use the ' + ' prescription to calculate the NLO contribution in $4+2 \epsilon$ dimensions. Instead they explicitly trace the cancellation of the singularities caused by the soft gluon emissions between the real and virtual contributions. Thus, it turns out, that the non-singlet structure function and the DGLAP evolution in the physical scheme are exactly the same as in the $\overline{\mathrm{MS}}$ scheme. Therefore the physical approach may be considered as an alternative factorisation scheme.

The online version of the original article can be found under doi:10.1140/epjc/s10052-013-2534-3.

a e-mail: emmanuel.de-oliveira@durham.ac.uk 